\title{
Anhang: Ausgewählte vollständige Texte
}

[ZJD_27.07.2006_Rau_001], E-Mail

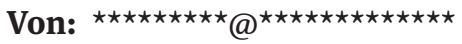

Gesendet: Donnerstag, 27. Juli 2006, 09:40

An: info@zentralratjuden.de

Betreff: mörderisches Verhalten

Sehr geehrte Damen und Herren,

da Sie sich dieser Tage ohne jede Kritik auf die Seite Israels stellen, ist nun wenigstens die Forderungen aus der Welt, man müsse zwischen dem Zentralrat und Israel differenzieren. Sie halten sich für eine Truppe, Sie sind eine Truppe.

Mit größter Empörung nehme ich wahr, daß Ihr zionistischer Staat zigtausende Menschen zu bombardieren und zu vertreiben, die nichts getan haben, außer dort zu leben, wo sich der seit 60 Jahren dauernde Kampf um eine gerechte Ordnung für die von Israel vertrieben Palästinenser abspielt. Fassungslos stelle ich fest, daß Ihnen tote Kinder in Gaza und Libanon offenbar gänzlich egal sind. Augenscheinliche vorsätzliche Tötung von UN-Soldaten gehört anscheinend auch zum Geschäft Ihres Staates. Herr Begin hat den Staat Israel mit Terror herbeigebombt und seither wird er mit Terror aufrecht erhalten.

Stellen wir uns einmal vor, dies alles täte Iran. Was wäre da los in Deutschland? Massendemonstrationen, Politiker, die harte Worte finden. Sie haben die öffentliche Meinung gut im Griff. Die Frau Bundesministerin für wirtschaftliche Zusammenarbeit wurde ja sofort abgemahnt, weil sie wagte, vorsichtig die Wahrheit zu formulieren. So kann das nicht weitergehen. Sie und der Staat Israel verhalten sich mit einer Frivolität, die ihresgleichen sucht. Moralisch unantastbar, im Besitz von Recht und Wahrheit. Hintergrund ist wohl die zionistische Ideologie, ein auserwähltes Volk zu sein. Wir Deutsche haben bittere Erfahrung mit Auserwählten, Sie müssen das wohl noch lernen. Statt in der sorgsam gepflegten Opferrolle immer mehr zum Täter zu werden, sollte Israel schnellstens erkennen, daß das durch seine Existenz geschaffene Unrecht an Tausendenden Palästinensern ausgeglichen werden muß, durch einen eigenen Staat ohne militante Siedlerinseln, durch Entschädigung für Eigentumsverlust, Jahrzehnte Lagerleben und die Chancenlosigkeit einer ganzen Generation und durch el Quds als Hauptstadt auch für diesen palästinensischen Staat. 
Existenzrecht kann nicht ein dauernd mit Gewalt zu verteidigender selbst gemachter Anspruch sein. Existenzrecht kann gerade in einem Fall, in dem ein Staat schlicht auf fremdem Territorium entstanden ist, nur bedeuten, den dadurch unvermeidbar verursachten Schaden der vorher dort lebenden Menschen auszugleichen. Machen Sie sich doch bitte klar, daß vor der Ankunft Israels im Nahen Osten Frieden herrschte. Seither ist Krieg. Gibt Ihnen das nicht irgendwie zu denken?

Mit freundlichen Grüßen

Prof. Dr. [Vorname Name]

[Adresse etc.]

[ZJD_01.09.2006_Tef_001], E-Mail

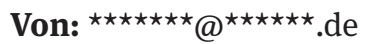

Gesendet: Freitag, 1. September 2006, 21:59

An: info@zentralratdjuden.de

Betreff: aktuelle Diskussion und Situation

Sehr verehrte Frau Knobloch, im Bewusstsein der Vergangenheit und der Verantwortung daraus, bin ich ein sensibilisierter Bürger dieses Landes, der die historische Verpflichtung aus der Geschichte in sich trägt und diese lebt.

Antisemitismus im Besonderen und der Ausgrenzung und Pauschalisierung gegenüber allen religiösen, ethnischen und individuellen Mitmenschen im Allgemeinen, bin ich gegenüber sehr sensibel und verpflichtet! Ich trage und leben die Verantwortung aus den Verbrechen der Deutschen zur Nazi-Zeit. Das ist meine Verpflichtung und tiefer, innerer Wert Deutscher zu sein. Gegenüber den Opfern der Ns-Diktatur fühle ich mich und bin ich verpflichtet

Sie und ihre Gemeinschaft werfen den Deutschen in den letzten Tagen Antisemitische- Stimmung und Tendenzen vor. Das stimmt meiner Meinung nach so nicht... und der Vorwurf darf so auch nicht gemacht werden. Für mich Das ist ein politischer, egoistischer Missbrauch eines ganz sensiblen Teils der Geschichte. Ich kann solche pauschalen Aussagen nicht nachvollziehen! In einer Demokratie mit mulit-religiösen Bürgern ist es mehr als normal seine Meinung auch äußern zu dürfen. Dazu gehören auch eine objektive Beurteilung der Vorgehensweise und Behandlung Israelis gegenüber seinen Nachbarn. 
Sie sollten das vielmehr wahrnehmen und beleuchten und nicht gleich mit dem Hammer verurteilen und schon gar nicht mit der Ausrede des Antisemitismus. Und schon gar nicht in Deutschland. da wir alle wissen was wirklicher Antisemitismus und die Folgen waren und sind ! Die Aussagen von Dieter Graumann :

„Unsere gemeinsame und wichtigste Aufgabe ist, die Verteidigung des Staates Israel und die dauerhafte Sicherung seiner Existenz in Frieden mit seinen Nachbarn. Wir werden unseren Teil dazu beitragen“, bekräftigte Graumann.

ist so nicht tragbar.

Wir sprechen vom Zentralrat der Juden in Deutschland -->von Deutschen. Deutschen Juden in Deutschland. Und NICHT von den Handlungen und Durchsetzung der israelischen Interessen und der Ablehnung jeder kritischen Stimmen

Ich erwarte vom Zentralrat eine ganz andere Haltung, Ausrichtung und Inhalte!

Ich muss Ihnen leider sagen dass Sie mit solchen Aussagen und Inhalten den Antisemitismus nur fördern, und den Antisemiten damit stärken. Ich hoffe wirklich dass die Das verstehen können. Ich höre und fühle was die Menschen denken und bewegt.

Was ich von Ihnen erwarte und auch vom Zentralrat allgemein ist folgendes:

Sie vertreten die Deutschen Juden. Nicht den Staat Israel. Deshalb würde ich mir eine objektive, kritischere Betrachtungsweise wünschen, zumindest aber die Akzeptanz und Wahrnehmung anderer Meinungen. Auch das ist eine historische Verpflichtung.

Juden in Deutschland dürfen nicht länger eine Gruppe von verfolgten, Unterdrückten und Opfern sein, sondern vielmehr fester Bestandteil der Gesellschaft mit den gleichen Rechten und Pflichten, wie auch alle anderen religiösen Gemeinschaften! Das sollten sie leben und vorantreiben Sorgen sie für gute gemeinschaftliche Verhältnisse, jüdisches Leben in Deutschland zu etablieren und zu stärken und nicht die Handlungen Israels zu rechtfertigen ; und schon gar nicht unter dem Vorwand des Antisemitismus wenn kritische Stimmen verlauten! Das ist kontraproduktiv. Ich bin wirklich sehr enttäuscht ! 
Ich lebe für Frieden und Freiheit, ein friedliches, brüderliches, kulturelles Miteinander, in den sich die religiösen und kulturellen Gruppen gegenseitig „befruchten“.

Wir müssen die guten Werte, Inhalte und gemeinsam leben. Das ist die richtige Entwicklung.

Ich sage es noch einmal. Das schilde ich auch den Opfern der Nazi-Herrschaft!

Mit den besten Grüßen und der Hoffnung auf eine Änderung ihrer Ausrichtung und Werte.

[Vorname Name]

[Adresse]

[IBD_20.07.2006_ano_008], E-Mail

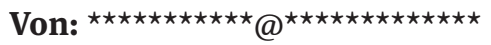

Gesendet: Donnerstag, 20. Juli 2006, 08:21:03 +0100

An: botschaft@israel.de

Betreff: Mörderwalze Israel

Habe soeben ein Interview mit Netanjahu in BBC4 gehört. Wenn ich diesem Herrn zu höre stellt sich mir die Frage:

„War Hitler ein weiser Mann-ein Mann der in die Zukunft schauen konnte?“

Ich kenne immer mehr Menschen die mit Hitlers kranker Idee Euch auszurotten sympathisieren. Ist das nicht beängstigend? DIese Leute behaupten sogar Ihr seid die neuen Nazis. Kann man dies nicht sogar verstehen?

Wenn N. dieMeinung von Euch Juden ist, ein Mensch der einen ganzen Korb von Unwahrheiten verbreitet gemixt mit Arroganz, Ignoranz, HerrenmenschenDenken.

Nur durch die internationale Community wurde Euer Krüppelstaat gegründet.

Die Welt wird von Euch an der Nase herumgeführt. Hunderte von Resolutionen wurden geblockt von US oder Ihr habt Euch einen S...... darum gekümmert. Warum habt Ihr heimlich Atombomben gebaut. Eure Nachbarn bezeichnet Ihr als Kriminelle. Mit einem Finger auf jemand anderen deuten heisst, das 3 Finger auf Euch zeigen.

Ich hoffe das die neue Deutsche Jugend sich von Eurem Jammerdiktat über die getöteten 6 oder 3? Millionen Juden löst und Euch zwingt sich den Regeln der Weltgemeinschaft anzupassen. DieEU sollte Euch als assoziiertes Mitglied 
mit Sanktionen belegen. Eure Geschichte ist voll von Blut. Täter seid Ihr nicht Opfer.

Ich hasse jegliche Gewalt auch Gewalt gegen Juden. Aber ich wünsche für die Zukunft meiner Kinder eine friedliche Welt. Ihr seid leider nicht daran interessiert, vielleicht wollt ihr ja die Araber ausrotten? Eurem kranken Gehirn fällt vielleicht sogar das ein!

[IBD_16.07.2006_Tsc_001], E-Mail

Von: ${ }^{\star \star \star \star \star \star \star \star \star \star \star \star \star \star ~ @ ~}{ }^{\star \star \star \star \star \star \star \star \star} . d e$

Gesendet: Sonntag, 16 Juli 2006, 10:12:23 +0200

An: botschaft@israel.de

Betreff: Kommentar zu den jüngsten Ereignissen im Libanon

Sehr geehrte Damen und Herren,

in den letzten Tagen bin ich mehr und mehr zu der Erkenntnis gelangt, dass der iranische Präsident Ahmadinedschad Recht hat: Die Welt wäre besser dran ohne die Existenz dieses aggressiven und unmenschlich handelnden zionistischen Gebildes in Palästina. Wieviele Menschenleben hätten seit 1948 gerettet werden können, wenn die UN niemals der Gründung dieses illegitimen zionistischen Gebildes in Palästina zugestimmt hätte?

Die jüngste Eskalation der Gewalt im Nahen Osten ist eindeutig die Schuld des zionistischen Gebildes: Denn die Angriffe der Hisbollah im Norden wurden erst durch das barbarische und menschenverachtende Vorgehen der zionistischen Militärmaschinerie im Gazastreifen sowie die Untätigkeit der Staatengemeinschaft, das zionistische Gebilde in die Schranken zu weisen, ausgelöst. Hätte sich das zionistische Gebilde besonnener und kompromissbereiter gezeigt, wäre der Konflikt mit der Hisbollah niemals ausgebrochen bzw. so eskaliert.

Aber wer die Geschichte des Nahostkonflikts kennt, der weiß, dass brutale Gewalt und Kollektivstrafen gegen ganze arabische Bevölkerungen (die übrigens laut Völkerrecht illegal sind) eine bewährte militärische Strategie des zionistischen Gebildes darstellen, von denen alle zionistischen Regierungen gerne Gebrauch gemacht haben. Scheinbar besitzt in der zionistischen 
Philosophie nur jüdisches Leben einen Wert, während tote arabische Zivilisten für das zionistische Gebilde stets moralisch in den Hintergrund traten.

Ebenso hat das illegitime zionistische Gebilde stets das Völkerrecht und die Appelle der internationalen Staatengemeinschaft ignoriert und missachtet. So auch jetzt im gegenwärtigen Konflikt. Mich persönlich verwundert es da nicht, dass die Araber keinen anderen Ausweg sehen, als sich in Einkaufspassagen des zionistischen Gebildes in die Luft zu sprengen. Wenn niemand auf der Welt den Palästinensern hilft, gibt es nur noch wenige Möglichkeiten zum Widerstand.

Langer Rede, kurzer Sinn: Ich finde, es ist an der Zeit, dass sich die zivilisierte Welt erhebt und endlich das sagt, was viele denken, aber nicht auszusprechen wagen: Die Errichtung des zionistischen Gebildes in Palästina gehört zu den großen Verbrechen der Menschheitsgeschichte, und das zionistische Gebilde genießt nicht das leiseste moralische Existenzrecht. Ich jedenfalls pflichte voll und ganz dem iranischen Präsidenten bei, wenn er sagt, der Zionismus sei eine Krankheit, und das zionistische Gebilde ein Unrechtsgebilde.

Ich hoffe, die jüdische Bevölkerung des zionistischen Gebildes begreift endlich, auf welchem moralischen Irrweg sie sich befindet, und wieviel Blut bereits jetzt an den Händen eines jeden jüdischen Israelis klebt. Es ist das Blut arabischer Kinder, Frauen und Männer, die seit 1948 durch das zionistische Gebilde zu Tode gekommen sind. Auch jetzt geht das zionistische Gebilde wieder eifrig seiner Hauptbeschäftigung nach: der Ermordung und Vertreibung arabischer Zivilisten. Es bleibt die Frage, ob sich das zionistische Gebilde damit nicht letztendlich selbst ins Bein schießt.

Mit freundlichen Grüßen

[Vorname Name] 
[IBD_10.08.2006_Uhl_001], Brief

Dr. [Vorname Name]

[Adresse]

D., den 10.08.2006

Herrn

Shimon Stein

Botschafter des Staates Israel

Auguste Viktoriastr. 74-76

14193 Berlin

Kriegsverbrechen Israelis im Libanon

Guten Tag, Herr Botschafter,

der von Ihnen vertretene Staat Israel hat mal wider alles getan um die Nachfolge Nazi-Deutschland unter Adolf Hitler nachzuahmen. Wie gleichen sich doch der Überfall Israels auf den Libanon mit seinen verherenden Zerstörungen der Infrastruktur und den fürchterlichen Folgen an den Menschen mit Hitlers Überfall auf Polen und Rußland. So hat die Entführung zweier israelischer Mordgesellen durch die Hisbollah bisher zu mehr als 1000 Toten, der Vernichtung ganzer Stadtteile und Häuser und zur Flucht von 500.000 Menschen geführt.

Der Judenstaat mit seinen heimatlosen Kosmopoliten, seinem Bombenholocaust und Staatsterrorismus ist jetzt endgültig zu einem Täterstaat und einem Tätervolk unter Olmert geworden.

Verbrechen gegen die Genfer-Konvention.

Israel tötet wahllose Zivilisten, greift Flüchtlingslager an, Phosphor und Streubomben sind Verbrechen gegen die Menschlichkeit und werden gegen die Bevöl- 
kerung eingesetzt. Hilfslieferungen des Roten Kreuzes und anderer Hilfsorganisationen werden behindert oder nicht durchgelassen. (Sollen die Menschen des Libanon auch“dünn“ gemacht werden so wie die Palästinenser im Gazastreifen?) Vergleiche mit dem Warschauer Ghetto drängen sich auf.)

Der Angriff auf Kana war angeblich ein Versehen. (Bester Beweis jüdischer Chuzpe!)

Die Kriegsverbrechend es Judenstaates in Sabra, Schattila und Dschenin waren sicher auch nur ein Versehen?

Die 250.000.000 Euro die der „begnadete“ ehemalige Außenminister der BRD (ehemals Scharons Schoßhündchen) dem Judenstaat, einschließlich des Geschenkes zweier U-Boote in den unersättlichen Rachen geworfen hat, sind sicher für Israels Kriegszüge gut angelegt worden. Den Bundesbürgern werden dafür die Steuern erhöht, daß die Bundesregierung in unerschütterlicherNibelungentreue zu Israel hält und seine Kriegsverbrechen finanziert. (1914 hat die Nibelungentreue Deutschlands zu seinen „Freunden“ zum 1.Weltkrieg geführt. Führt Herrn Olmerts Wunsch, sich mit deutscher Schutztruppe zu schützen, in dieser Gemengelage wohlmöglich zum 3.Weltkrieg?)

Ich hoffe, daß die Kämpfer der Hisbollah dem blindwütigen Treiben Israels bald ein Ende setzen und unsere Partei-Nullen in Berlin endlich eine distanzierte Haltung zum Judenstaat bekommen.

Fazit: Der Judenstaat Israel gleicht im Moment einer stinkenden Pestbeule, die ausgetrocknet werden muß.

Vorschlag: Die Herren Olmert und Perez sind als Kriegsverbrecher international zur Fahndung auszuschreiben und dem Kriegsverbrecher-Tribunal in den Haag zu überstellen.

Es grüßt Sie

Dr. [Vorname Name] 
[ZJD_04.08.2006_Gra_001], E-Mail

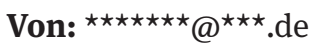

Gesendet: Freitag, 4. August 2006, 13:14

An:info@zentralratdjuden.de

Betreff: aufforderung

Sehr geehrte Frau Knoblauch.

Was sich in den letzten drei Wochen im Mittleren Osten ereignet, ist ausschliesslich den Juden zuzuschreiben. Unterstuetzt von juedischen Geldgebern aus London und Amerika versuchen sie die totale Kontrolle ueber die Araber zu gewinnen, damit Ihre Geldgeber an die Oelquellen kommen und somit ihr schmutziges Geldschiebergeschaeft, was die Juden bisher immer schon getan haben. fortzusetzen. Bush und Blair sind doch nur Handlanger. Kennen Sie die Information in dem Buch „,Die Absteiger““. Einen Herrn Murdoch hat die australische Regierung ausgewiesen. Zieht man Bilanz auf Ihr Volk, so steht es nicht anderes wie mit Zigeunern. Ihre Mitglieder haben sich in der ganzen Welt herumgetrieben und fordern, was ihnen nicht zusteht. Wo sind die geklauten Gelder eines Herrn Maier aus Frankfurt? Dieser hatte eine Bank in Frankfurt; aenderte spaeter seinen Namen in Rot Schild, indem er ein solches Schild ueber seine Bank haengte. Verschwand dann sehr schnell mit Geldeinlagen und finazierte den Krieg zwischen England und Frankreich, wo er riesige Summen verdiente, bis er sich dann nach Amerika absetzte und dort die Rotschildbank gruendete. Das Buch die "Absteiger“ zeigt unverfaelschte Dokumente ueber den eigentlichen Verlauf der Entwicklung der Juden.

Sie sollten besser daran tun, Ihrer Regierung zu empfehlen, dass der Hass gegen Ihr Volk nicht zunimmt. Alle diese von Ihren Publizisten und Medienbossen verbreiteten Meldungen basieren auf Falschmeldungen und Irrefuehrungen. Sie muessen sich eingestehen, dass Ihre Mitglieder, egal wo sie leben, nur Gaeste sind. Sie haben keinen Bundesstaat und somit- wie schon frueher - versuchen Sie sich ueberall einzuschleichen, um Ihre Macht auszudehnen. Die Zeit scheint aber abzulaufen.

Ich erwarte Ihre Nachricht und werde, was vollkommen legal ist, Details aus weiteren orginal Dokumenten publizieren.

Mit freundlichen Gruessen

[Vorname Name] 
[IBD_28.07.2006_Pfa_001], Brief

[Dr. Vorname Name]

[Adresse] 28-07-06

Exzellenz,

Die Vorgänge im Nahen Osten beunruhigen mich. Das Verhalten der Israelischen Seite halte ich für völlig unangemessen und schlichtweg verbrecherisch. Der in Gaza „gekidnapte“ Soldat hatte immerhin den Auftrag zu einer kriegerischen Handlung.

Wird Herzl's 1860 Traum vom „Judenstaat“ (1903: „wenn ihr es wollt, ist es kein Märchen“) nun zum Alptraum?

Oder wie zeitnah erscheint auf einmal:

Numeri 33.50-52 Und der Herr sprach zu Moses. „Rede mit den Söhnen Israels .... Zieht .. in das Land..., dann müßt ihr die Insassen des Landes vor euch vertilgen. Auch .. sollt ihr .... all ihre Höhen verwüsten. Vor Land ergreift Besitz und siedelt darin! Denn Euch gebe ich das Land zum Besitz.“ Der Herrgott als Vollstrecker Lord Balfours? Wollen die derzeitigen Machthaber den mehrere Tausend Jahre alten Auftrag perfektionieren?

Warum sollen eigentlich, nach der, dem UN-Beschluss entgegenstehenden, „Staatsgründung 48“ durch Ben Gurion (früher David Grün) (nur) die Palästinenser den Preis (z. B. für den Holocaust) bezahlen?

In Palästina waren ursprünglich ca. 5 Mio Muslime und ca. 400000 Juden. Heute sind die Palästinenser verjagt, (weil sie die kriegerische Auseinandersetzung verloren hatten) und das Zahlenverhältnis ist umgekehrt. Zunächst (David Grün setzte sich besonders dafür ein) kamen, wenn es ihnen gelang, die Flüchtlinge vor der Naziherrschaft. Die Engländer taten alles, um den Zustrom in ihr Mandatsgebiet zu verhindern. Dann wurden etwas später, damit man genügend Volk hatte, aus aller Welt Menschen angeheuert, ins Land zu kommen. (zuletzt meinten Palästinenser aus Gaze, daß etliche Soldaten nicht einmal richtig Hebräisch können.) 
Der „Held von sabra und shantilla“, auch „Vater der Siedlungsbewegung“ genannt, das bezog sich damals auf Samaria, Judäa, Gaza, war auf einmal für den Rückzug der Siedler aus dem Gazastreifen. Nun, für Gaza brauchte man für den Schutz vier mal so viele Soldaten wie Siedler. Wurde für den Rückzug eine nüchterne Finanzbetrachtung zum ehrenwerten Motiv umgemünzt?

Saddam Hussein wurde vorgeworfen, er unterstütze antiisraelische Kräfte.

Als Gamal Abd El Naser 1956 den Suezkanal verstaatlicht, überfällt im Okt 56 Israel Ägypten, dann greifen Engländer und Franzosen ein.

Wer hat die Flugzeuge bereit gestellt, die (von einem israelischen Collonel vor fast 40 Jahren selber gehört) für den Krieg 67 schnellstens umgespritzt werden mußten, damit die Erkennungszeichen verschwanden. Wer liefert, laut Zeitungsbericht die 3 Mrd \$ Militärhilfe, wer liefert im Rahmen einer militärischen Unterstützung die ca. 5000 Bomben von denen 500 bis zu 3m Beton durchbrechen? Wo sind die Ziele mit 3m Beton? Wie geschäftstüchtig sind die israelischen Verhandler, wenn Deutschland die U-Boote mit ca 30\% Preisnachlaß liefert? Übrigens an Taiwan nicht liefern durfte, weil es sich um Krisengebiet handelt. Fragen, die einen nachdenklich machen können.

An einen ganz schlimmen Spruch wurde ich gerade aus gegebenem Anlaß erinnert: -- Hitler: im großdeutschen Rundfunk mitgehört: „für einen getöteten Deutschen 100 Geiseln“ - (Manches brennt sich einem ein, auch die Gestapo (zum ersten Mal) 1938. Ein „gekidnapter“ Israeli gegen ein Kraftwerk? Die Verhältnismäßigkeit für entführte Soldaten. So schlimm das ist. Israel durfte zwar beliebig, wie man immer wieder in der Presse lesen konnte, Menschen verhaften und jahrelang ohne Prozeß einsperren, notfalls sie auch vom Helikopter aus mit Raketen umbringen. Auch wenn da noch ein paar Zivilisten umkamen. Übrigens, war dieser Soldat in bei einem von Israel befohlenen kriegerischen Einsatz, als er festgenommen wurde.

Und noch etwas: Vor 1945 haben wir von Sippenhaft gesprochen. Wie ist das, wenn man das Haus der Familie eines Selbstmordattentäters ausbombt? Alttestamentarisches Zahn um Zahn?

Und weil ich gerade auf dieser Welle bin: Bezüglich des von der Knesset verabschiedeten Ehegesetzes wundere ich mich, wie schüchtern sich die USA verhalten, die doch bei Südafrika noch so kühn aufgetreten sind. --- obwohl sie (nicht nur davor) noch genügend eigene Probleme hatten. 
Wer die Situation mit einigermaßen wirtschaftlichem Verstand (aus den Wirtschaftsteilen der Zeitungen abzuleiten) beobachtet, kann nachrechnen, daß Israel aus dem eigenen Bruttosozialprodukt nicht in der Lage wäre, die fortwährende Situation, ohne von außen kommende außerordentliche Unterstützung zu beherrschen. Woher kommt die? Übrigens um 1986 wurde Irak für den Stellvertreterkrieg gegen Iran unterstützt. Haben die USA mit ihrer Unterstützung etwa vielleicht eigene Ziele? Vergleich mit 1956 Suez?

Gerade jetzt wurden die nachdenklichen Bemerkungen von Kofi Annan im Fernsehen vorgetragen, aber unmittelbar darauf wurde er durch das Auftreten eines Amerikaners geradezu lächerlich gemacht. USA-Politik? Und für einen Israelischen General sind, wie er im Interview meinte, die Entscheidungen und Empfehlungen der UN irrelevant.

Ganz katastrophal die Bemerkung eines Israelischen Generals, man wolle Libanon um 20 Jahre zurückbomben.

Es ist schon auffallend, wie Israel UN-Resolutionen einfach überhaupt nicht beachtet, ohne, daß das irgendwelche Folgen hätte. Im Zweifelsfall legt der USAVertreter sein Veto ein, was den Verdacht einer gewissen Parteilichkeit aufkommen läßt, und die Sache nur schlimmer macht. Geld- Material- und Waffentransfer waren schon angesprochen. Stimmt es, daß in den USA ungefähr ebenso viele Juden leben, wie in Israel.

Allewelt scheint sich darüber einig zu sein, daß die Hisbollah oder Hamas die Bösen sind. Die Irgun oder auch Haganna - von den Engländern so gefürchtet, daß die schließlich sogar abzogen - waren anscheinend die Guten? Ich erinnere mich noch an die Radioberichte über den Bombenanschlag auf die britische Botschaft in Rom 1946. Primitive Frage: die erstere Gruppe kämpfte um ihr Land um es zu behalten, die zweite Gruppe um es sich anzueignen (Numeri?). Jüdische Siedlungen wie Sommersprossen auf der Landkarte eines Landes, das bisher anderen gehörte. Ist etwa der UNO-Beschluß von vor 1948 von Israel beachtet worden, oder hat Ben Gurion eigenmächtig gehandelt?

Wenn heute die überwiegende Mehrheit der Israelischen Gesellschaft (entsprechend einseitig und im Zweifelsfall sogar betrügerisch indoktriniert) für diese erneute kriegerische Auseinandersetzung bejaht, dann erinnere ich mich das an den Berliner Sportpalast: „wollt ihr den totalen Krieg“. Welcher Göbbels hat es denn jetzt den Israelis indoktriniert? 
Es gibt ja so viel, über das man nachdenklich werden muß. Matthäus 27.24 „Ich bin unschuldig am Blute dieses Gerechten“ und die Menge schrie: 27.25: „Sein Blut komme über uns und unsere Kinder.“ Das hatte schlimmes Fehlverhalten zur Folge.

Und vielleicht war es ja gar nicht nur die Tempelzerstörung und Kaiser Hadrian mit der Vertreibung der Juden aus Jerusalem. Vielleicht war es eher der abtrünnige Paulus, der dem Jüdischen Volk seine Identität raubte. Luc. 4.14 1. Brief an die Thesaloniker. Die Diaspora der Juden? ... die dann 2000 Jahre später (vielleicht sogar ein Missverständnis in der eigenen Religion) in „ihr“ Land zurückkehren?

Kaiser Konstantin verlangte von den Juden eine Extrasteuer. Es gab Progrome in Spanien, in 1348 Prag die Judenschlacht, die Progrome in Rußland und Rumänien, auf die sich Herzl bezog, es gab den Terror in Deutschland, es gab, was BenGurion bewegte, zu helfen, es gab den 9. November 1938, und es gab Maidaneck, Birkenau, Treblinka Theresienstadt, Auschwitz und so weiter und es gab über 6 Mio Umgebrachte.

Genügt da ein Lea Rosh Gedächtnisfeld? Und wie verhalten sich die Enkel?

Der Antisemitismus hätte (vielleicht auf einer Linie wie Barenboim, Seligmann, vielleicht auch Ihres Vorgängers Avi Primor) vorbei sein können. Zeigen die Handlanger (nicht nur in Israel), wie man mit alttestamentarischer Rachsucht nicht den Frieden erreichen kann sondern den Antisemitismus fördert?

Sie Herr Botschafter müssen dabei selbstverständlich dem Auftrag Ihrer Regierung nachkommen.

Ich gehe davon aus, daß eine Botschaft als Informationsquelle für ihr Land dient. In diesem Sinne möchte ich Ihnen und Ihrer Regierungsmannschaft meine Meinung übermitteln. Es ist schlichtweg unakzeptabel, wie einer der israelischen „Vollstreckern des Bösen“ in typisch menschenverachtender Weise gemeint hat, den Libanon um 20 Jahre zurückzubomben. Ist das Israel? Sind das die $80-90 \%$ Israelis, die für den Krieg sind? Schon 1982 hatte Sharon versprochen, den Terrorismus ein- für allemal beendigt zu haben. Na und...? Und wer belügt das Volk heute?

Die USA wollen sich, wie jetzt angekündigt, vorläufig zurückhalten, (damit die von USA gelieferten F16 in Stil eines Bomber-,Harris“ (in Deutschland wissen wir, was Bombenterror bedeutet) noch mehr niederbomben können?), dann erin- 
nert mich das erschreckend an „Belehrungen“ aus 1943, die uns vor der „Bedrohung durch das Internationale Judentum“ (Mein Kampf) warnten. (an Menschen zahlengleich in USA und Israel? US-Militärhilfe 3 Mrd \$?, umgespritzte US-Flugzeuge?, 5000 Tausend US-Präzisionsbomben?, wiederholtes Veto des US-Vertreters bei der UN, wenn es um Kritik an Israel ging? Sharon war häufiger als jeder andere Präsident zu Besuch beim US-Präsident).

Es könnte der Eindruck entstehen, Israel habe nie wirklich Frieden gewollt. Jeder Rabbatz von der anderen Seite schien mehr als erwünscht. Den Gegner so lange provozieren, bis er endlich zuschlägt. Damit hatte man die Rechtfertigung zur Gegenmaßnahme. Ansonsten mußte Sharon im September 2000 erst mal provokativ auf dem Tempelberg auftreten. Und mit der damit ausgelösten Intifada sah man, wo die Bösen sind?

Das ganze Dilemma ist dadurch entstanden, daß Israel von Anfang an allezeit eigenwillig und überheblich geglaubt hat, sich selber durchzusetzen. Man hatte geglaubt, so mehr zu erreichen, was schert uns UNO, als in Zusammenarbeit mit den anderen Staaten. Israel hat nicht kapiert, daß es nichts bringt, Selbstmordattentätern mit der Todesstrafe zu drohen.

Ein kräftiger Beitrag zu einem internationalen Antisemitismus wird durch die maßlose Verhaltensweise Israels, mit dem Verbrechen an den Menschen in der Nachbarschaft mit Sicherheit erreicht werden. Die einseitige Unterstützung durch die USA zeigt die Verlogenheit der internationalen Politik.

mit freundlichem Gruß

Dr. [Vorname Name]

[IBD_19.04.2008_Bec_001], E-Mail

Von: ${ }^{\star \star \star \star \star \star \star \star \star \star ~ @ ~}{ }^{\star \star \star}$.de

Gesendet: Samstag, 19. April, 2008, 17:01

An: botschaft@israel.de

Betreff: Ihr Besuch bei uns an der Schule

Sehr geehrter Herr Mor,

Sie waren bei uns in der Schule, um für Israel zu werben. 
Wenn alle Juden so sind, wie Sie, dann wissen wir jetzt warum die Vergangenheit in Deutschland so passiert ist.

Die meisten aus unserem Kurs fanden Sie genauso abstoßend wie ich. Vielen Dank, dass Sie uns Schülern in Ihrer Person die Erkenntnis gebracht haben, wie Juden wirklich sind.

Mit freundlichen Grüßen

P. S.: dies ist die Email-Adresse von einer Freundin, sie würden ja sonst zur Schule petzen gehen..., wie die Juden es immer tun. 